\title{
A construction of small regular bipartite graphs of girth 8
}

\author{
Camino Balbuena $\rrbracket^{\natural}$ \\ ${ }^{1}$ Departament de Matemàtica Aplicada III, Universitat Politècnica de Catalunya, Campus Nord, Jordi Girona 1 i 3 , \\ 08034Barcelona, Spain
}

received September 6, 2007, revised January 22, 2009, accepted June 25, 2009.

Let $q$ be a prime a power and $k$ an integer such that $3 \leq k \leq q$. In this paper we present a method using Latin squares to construct adjacency matrices of $k$-regular bipartite graphs of girth 8 on $2\left(k q^{2}-q\right)$ vertices. Some of these graphs have the smallest number of vertices among the known regular graphs with girth 8 .

Keywords: Incidence matrices, Latin squares, cages, girth.

\section{Introduction}

Throughout this paper, only undirected simple graphs without loops or multiple edges are considered. Unless otherwise stated, we follow the books by Godsil and Royle [16] and by Lint and Wilson [21] for terminology and definitions.

Let $G=(V(G), E(G))$ be a graph with vertex set $V=V(G)$ and edge set $E=E(G)$. The girth of a graph $G$ is the number $g=g(G)$ of edges in a smallest cycle. The degree of a vertex $v \in V$ is the number of vertices adjacent to $v$. A graph is called regular if all the vertices have the same degree. A cage is a $k$-regular graph with girth $g$ having the smallest possible number of vertices. Simply counting the numbers of vertices in the distance partition with respect to a vertex yields a lower bound $n_{0}(k, g)$ on the number of vertices $n(k, g)$ in a cage, with the precise form of the bound depending on whether $g$ is even or odd.

$$
n_{0}(k, g)= \begin{cases}1+k+k(k-1)+\ldots+k(k-1)^{(g-3) / 2} & \text { if } g \text { is odd; } \\ 2\left(1+(k-1)+\ldots+(k-1)^{g / 2-1}\right) & \text { if } g \text { is even }\end{cases}
$$

As defined by Biggs [7], the excess of a $k$-regular graph $G$ is the difference $|V(G)|-n_{0}(k, g)$. A $(k, g)$-cage with even girth $g$ and $n_{0}(k, g)$ vertices is said to be a generalized polygon graph. Generalized polygon graphs exist if and only if $g \in\{4,6,8,12\}$ [7]. The question of the construction of graphs with small excess is a difficult one. When $g=6$, the existence of a graph with $n_{0}(k, 6)=2\left(k^{2}-k+1\right)$

\footnotetext{
$\dagger^{T}$ This research was supported by the Ministry of Science and Innovation, Spain, and the European Regional Development Fund (ERDF) under project MTM2008-06620-C03-02/MTM.
}

1365-8050 @ 2009 Discrete Mathematics and Theoretical Computer Science (DMTCS), Nancy, France 
vertices called generalized triangle, is equivalent to the existence of a projective plane of order $k-1$, that is, a symmetric $\left(\left(n_{0}(k, 6)\right) / 2, k, 1\right)$-design. It is known that these designs exist whenever $k-1$ is a prime power, but the existence question for other values remains unsettled. Generalized quadrangles when $g=8$, and generalized hexagons when $g=12$ are also known to exist for all prime power values of $k-1$ [5, 16, 21].

Cages have been studied intensely since they were introduced by Tutte [27] in 1947. Erdôs and Sachs [10] proved the existence of a graph for any value of the regularity $k$ and the girth $g$, thus most of work carried out has been focused on constructing a smallest such graph $[1,2,4,6,9,11,13,15,22,23,24$, 28, 29]. Biggs is the author of an impressive report on distinct methods for constructing cubic cages [8]. For some time, Royle [26] kept a web-site in which all the cages known so far appear. More details about constructions on cages can be found in the survey by Wong [29], the survey by Holton and Sheehan [17] or on the more recent dynamic cage survey by Exoo and Jajcay [12].

It is conjectured that cages with even girth are bipartite [25, 29]. A graph is bipartite if its vertex set $V$ can be partitioned into two partite sets, $V_{1}$ and $V_{2}$, such that any edge has one end in $V_{1}$ and the other in $V_{2}$. If the vertices are ordered in such a way that the vertices of $V_{1}$ come first, then the adjacency matrix of a bipartite graph can be written in the form

$$
A=\left(\begin{array}{ll}
0 & N \\
N^{\top} & 0
\end{array}\right) .
$$

An incidence graph is a bipartite graph in which the elements of one part $V_{1}$ are declared as lines and the elements of the other part $V_{2}$ are declared as points. The terminology for incidence graphs is geometric. A point and a line are said to be incident if they are adjacent, thus the submatrix $N$ of (2) is called an incidence matrix of the bipartite graph. If the number of points and the number of lines coincide, then $N$ is clearly a square matrix. An incidence matrix $N$ defines a partial plane when

- any line has at least two points, and

- two points are incident with at most one line.

Consequently, two lines of a partial plane have at most one point in common. The corresponding bipartite graph is called the incidence graph of the partial plane, which clearly has even girth $g \geq 6$. Thus for simplicity we shall say that the partial plane has girth $g / 2$ if and only if the corresponding incidence graph has girth $g$.

Let $q$ be a prime power and $k$ an integer such that $3 \leq k \leq q$. In [3], incidence matrices of $(k, 6)$ bipartite graphs of order $2(k q-1)$ were given. In this paper we present a method to construct the incidence matrices of $k$-regular bipartite graphs of girth $g=8$ on $2 q(k q-1)$ vertices.

\section{Position matrices}

Let $S$ denote a set of symbols and let $A$ be a matrix whose elements are subsets of $S$. Given $x \in S$ let $P_{x}(A)$ be a $(0,1)$-matrix of the same dimension as $A$ that satisfies

$$
\left(P_{x}(A)\right)_{i j}=1 \text { if and only if } x \in A_{i j} .
$$

Thus, $P_{x}(A)$ is called the position matrix of the symbol $x$ in $A$. Suppose that $S=\left\{0, x_{1}, \ldots, x_{n}\right\}$. The position matrices of all the symbols in $A$ different from 0 give rise to the following $(0,1)$-matrix $\mathcal{P}(A)$ 
called position matrix of A:

$$
[\mathcal{P}(A)]=\left[P_{x_{1}}(A) \cdots P_{x_{n}}(A)\right] .
$$

Let $\left\{A^{1}, A^{2}, \ldots, A^{r}\right\}$ be a family of matrices of the same number of columns whose elements are subsets of $S$. Then the $(0,1)$-matrix spanned by the position matrices of all of them

$$
\left(\begin{array}{c}
\mathcal{P}\left(A^{1}\right) \\
\mathcal{P}\left(A^{2}\right) \\
\vdots \\
\mathcal{P}\left(A^{r}\right)
\end{array}\right)=\left(\begin{array}{ccc}
P_{x_{1}}\left(A^{1}\right) & \cdots & P_{x_{n}}\left(A^{1}\right) \\
P_{x_{1}}\left(A^{2}\right) & \cdots & P_{x_{n}}\left(A^{2}\right) \\
& \vdots & \\
P_{x_{1}}\left(A^{r}\right) & \cdots & P_{x_{n}}\left(A^{r}\right)
\end{array}\right)
$$

is said to be the position matrix of the family $\mathcal{F}=\left\{A^{1}, A^{2}, \ldots, A^{r}\right\}$. The following example shows two matrices of order $2 \times 2$ whose elements are subsets of $S=\{0, a, b\}$ and the position matrix of them. From now on, if there is no confusion the 1 -sets will be indicated as integers.

\begin{tabular}{|c|cc||cc|cc|}
\hline \multicolumn{2}{|c|}{ MATRICES } & \multicolumn{3}{|c|}{ SYMBOLS } \\
\cline { 4 - 6 } \multicolumn{2}{|c|}{} & & $a$ & & $b$ \\
\hline \hline$A^{1}$ & $a$ & $a$ & $\mathbf{1}$ & $\mathbf{1}$ & 0 & 0 \\
& $b$ & $b$ & 0 & 0 & $\mathbf{1}$ & $\mathbf{1}$ \\
\hline$A^{2}$ & $\{a, b\}$ & 0 & $\mathbf{1}$ & 0 & $\mathbf{1}$ & 0 \\
& 0 & $\{a, b\}$ & 0 & $\mathbf{1}$ & 0 & $\mathbf{1}$ \\
\hline
\end{tabular}

As already mentioned in the Introduction, our main aim is to obtain incidence matrices of bipartite $k$-regular graphs of girth 8 with small excess. Such incidence matrices may be seen also as incidence matrices of partial planes which will be obtained by identifying row $i$ of $P_{z}\left(A^{\alpha}\right)$ as line $i(\alpha)$, and column $j$ of $P_{z}\left(A^{\alpha}\right)$ as point $j(z)$, for any matrix $A^{\alpha} \in \mathcal{F}$ and $z \in S-0$. To achieve our goal we propose the following definitions.

Definition 2.1 Let $g \geq 4$ be an even number. A family of matrices $\mathcal{F}=\left\{A^{1}, A^{2}, \ldots, A^{r}\right\}$ of the same number of columns whose elements are subsets of a set of symbols $S$ is said to have girth $g$ if the position matrix of $\mathcal{F}$ is the incidence matrix of a bipartite graph of girth $g$.

Each matrix of 4 has girth $g=\infty$, and the two matrices $A^{1}, A^{2}$ form a family of girth 8 .

Let us recall that a Latin square of order $q$ is a $q \times q$ matrix with entries from a set of $q$ symbols such that each symbol occurs exactly once in each row and exactly once in each column. A Latin square has clearly girth $g=\infty$ because the position matrices of its elements are permutation matrices yielding the incidence matrix of a partial plane consisting in a set of parallel lines (since they have no common point).

In [3] we introduced the notion of quasi row-disjoint matrices as follows.

Definition 2.2 [3] Let $A^{1}$ and $A^{2}$ be two matrices of the same number of columns whose elements are subsets of a set of symbols $S$ such that $0 \in S$. A pair $(x, y)$ with $x, y \in S$ belongs to the cartesian product of any two rows $\left(A^{1}\right)_{i} \times\left(A^{2}\right)_{h}$ if and only if $(x, y) \in\left(A^{1}\right)_{i j} \times\left(A^{2}\right)_{h j}$ for some $j$. Then $A^{1}$ and $A^{2}$ are said to be quasi row-disjoint if and only if the cartesian product of any two rows $\left(A^{1}\right)_{i},\left(A^{2}\right)_{h}$ contains at most one pair $(x, x) \in\left(A^{1}\right)_{i} \times\left(A^{2}\right)_{h}$ with $x \neq 0$. 
The pair of matrices of the example (4) are quasi row-disjoint matrices. Moreover, in [3] we have stated the following theorem which roughly speaking says that a family of $r$ matrices is quasi row-disjoint if and only if its girth is at least 6 .

Theorem 2.1 [3] Let $A^{1}$ and $A^{2}$ be two matrices each one of girth at least 6 of the same number of columns and whose elements are subsets of a set of symbols $S$ such that $0 \in S$. Then $A^{1}$ and $A^{2}$ are quasi row-disjoint if and only the family $\left\{A^{1}, A^{2}\right\}$ has girth at least 6 .

In the next theorem we give a sufficient condition for a family of matrices to have girth at least 8 .

Theorem 2.2 Let $\mathcal{F}=\left\{A^{1}, \ldots, A^{r}\right\}$ be a set of $r \geq 2$ quasi row-disjoint matrices of the same number of columns whose elements are subsets of a set of symbols $S$ such that $0 \in S$. Let $\left(A^{u}\right)_{i},\left(A^{v}\right)_{i^{\prime}},\left(A^{w}\right)_{i^{\prime \prime}}$ denote any three mutually distinct rows of matrices $A^{u}, A^{v}, A^{w} \in \mathcal{F}$. Then the girth of $\mathcal{F}$ is at least 8 if the sets $\left(A^{u}\right)_{i} \times\left(A^{v}\right)_{i^{\prime}},\left(A^{v}\right)_{i^{\prime}} \times\left(A^{w}\right)_{i^{\prime \prime}},\left(A^{u}\right)_{i} \times\left(A^{w}\right)_{i^{\prime \prime}}$ contains at most two distinct pairs $(x, x)$, $(y, y)$ with $x, y \neq 0, x, y \in S$.

Proof: Suppose $(x, x) \in\left(A^{u}\right)_{i} \times\left(A^{v}\right)_{i^{\prime}}, x \neq 0$. Therefore the position matrix of $\mathcal{F}$ has the following entries equal to 1 :

$$
P_{x}\left(A^{u}\right)(i, j)=P_{x}\left(A^{v}\right)\left(i^{\prime}, j\right)=1,
$$

where $P_{x}\left(A^{u}\right)$ and $P_{x}\left(A^{v}\right)$ are the position matrix of the element $x$ in $A^{u}$ and $A^{v}$ respectively. Recall that for any given matrix $A^{\alpha} \in \mathcal{F}$ and $z \in S-0$, row $i$ of $P_{z}\left(A^{\alpha}\right)$ is line $i(\alpha)$, and column $j$ of $P_{z}\left(A^{\alpha}\right)$ is point $j(z)$. Consequently, (5) means that lines $i(u)$ and $i^{\prime}(v)$ have point $j(x)$ as a common point.

Analogously, $(y, y) \in\left(A^{v}\right)_{i^{\prime}} \times\left(A^{w}\right)_{i^{\prime \prime}}, y \neq 0$, is equivalent to:

$$
P_{y}\left(A^{v}\right)\left(i^{\prime}, j^{\prime}\right)=P_{y}\left(A^{w}\right)\left(i^{\prime \prime}, j^{\prime}\right)=1,
$$

or in other words, lines $i^{\prime}(v)$ and $i^{\prime \prime}(w)$ have the point $j^{\prime}(y)$ in common, with $j^{\prime}(y) \neq j(x)$ because $y \neq x$. Thus if there exists $z \neq 0, z \neq x, y$, such that $(z, z) \in\left(A^{u}\right)_{i} \times\left(A^{w}\right)_{i^{\prime \prime}}$, then lines $i(u)$ and $i^{\prime \prime}(w)$ have the point $j^{\prime \prime}(z)$ in common, $j^{\prime \prime} \neq j, j^{\prime}$, yielding that the partial plane defined by the position matrix of $\mathcal{F}$ contains the triangle $j(x) j^{\prime}(y) j^{\prime \prime}(z)$. In other words, the position matrix of $\mathcal{F}$ is the incidence matrix of a bipartite graph of girth less than 8 .

Our immediate goal is to derive a method for constructing a family of matrices with girth 8 , because the position matrix of this family will be the incidence matrix of a bipartite graph of girth 8 .

\section{Method}

Throughout this work let $[[n]]$ denote the set of non negative integers $\{0,1, \ldots, n\}$ and $(n]]=[[n]] \backslash\{0\}$. Let $I_{n}$ be the identity matrix and denote by $(t \times F) I_{n}$ the matrix obtained from $I_{n}$ by replacing each one with a subset $\{t\} \times F$ of $\{t\} \times[[n]]$ for some $t \in[[n]]$ and $F \subseteq[[n]]$. In the following theorem we demonstrate a method for obtaining a family of matrices with girth 8 using Latin squares.

Theorem 3.1 Let $q$ be a prime power, and let $\mathbb{F}_{q}$ be the Galois field of order $q$. For each $u, t \in \mathbb{F}_{q}$, define the $q \times q$ matrix $L^{u, t}$ by

$$
L^{u, t}(i, j)=i+u j+u t, i, j \in \mathbb{F}_{q} .
$$

Then the following assertions hold: 
(i) For all $u, v \in \mathbb{F}_{q} \backslash\{0\}, u \neq v$, and $t, t^{\prime} \in \mathbb{F}_{q}$ the matrices $L^{u, t}$ and $L^{v, t^{\prime}}$ are quasi row-disjoint Latin squares with entries from $[[q-1]]$.

(ii) For any given $u, t \in \mathbb{F}_{q}, u \neq 0$, the matrix $L^{u, t} \times L^{u+u^{-1}, t}$ has $q^{2}$ distinct entries. Moreover, the position matrix of the family

$$
\left\{L^{u, t} \times L^{u+u^{-1}, t}: t \in \mathbb{F}_{q}\right\}
$$

is a $(0,1)$-matrix of order $q^{2} \times q^{3}$ considering symbol $(0,0)$ different from 0 , and it is the incidence matrix of a partial plane consisting in $q^{2}$ parallel lines with q points on each.

(iii) The family $\left\{L^{u, t} \times L^{u+u^{-1}, t}: u, t \in \mathbb{F}_{q}, u \neq 0\right\}$ has girth 8 and its position matrix has order $\left(q^{3}-q^{2}\right) \times q^{3}$ and $q$ ones in each row and $q-1$ ones in each column.

(iv) The position matrix of the family

$$
\left\{L^{u, t} \times L^{u+u^{-1}, t}: u, t \in \mathbb{F}_{q}, u \neq 0\right\} \cup\left\{(t \times[[q-1]]) I_{q}: t \in \mathbb{F}_{q}\right\}
$$

is the incidence matrix of a q-regular bipartite graph of girth 8 with $q^{3}$ vertices in each partite set.

Proof: (i) Clearly $L^{u, t}$ and $L^{v, t^{\prime}}$ are $q \times q$ Latin squares on $[[q-1]]$. Let us show that they are quasi row-disjoint. Otherwise, there exists $i, i^{\prime} j, j^{\prime} \in \mathbb{F}_{q}$ with $j \neq j^{\prime}$ such that

$$
\begin{aligned}
& L^{u, t}(i, j)=L^{v, t^{\prime}}\left(i^{\prime}, j\right), \\
& L^{u, t}\left(i, j^{\prime}\right)=L^{v, t^{\prime}}\left(i^{\prime}, j^{\prime}\right) .
\end{aligned}
$$

Equivalently,

$$
\begin{aligned}
& i+u j+u t=i^{\prime}+v j+v t^{\prime} \\
& i+u j^{\prime}+u t=i^{\prime}+v j^{\prime}+v t^{\prime}
\end{aligned}
$$

Therefore $u\left(j-j^{\prime}\right)=v\left(j-j^{\prime}\right)$ implying that $u=v$ or $j=j^{\prime}$, a contradiction in either case.

ii) Note that $L^{u+u^{-1}, t}$ is also a Latin square if $u+u^{-1} \neq 0$, otherwise $L^{0, t}(i, j)=i$. In either case it is very easy to check that $L^{u, t}$ and $L^{u+u^{-1}, t}$ are orthogonal, which implies that $L^{u, t} \times L^{u+u^{-1}, t}$ has $q^{2}$ distinct entries. Hence, the position matrix of the family $\left\{L^{u, t} \times L^{u+u^{-1}, t}: t \in \mathbb{F}_{q}\right\}$ is a $(0,1)$-matrix of order $q^{2} \times q^{3}$ by considering entry $(0,0)$ different from 0 .

Let us show now that for every $i, i^{\prime}, j, t, t^{\prime} \in \mathbb{F}_{q}$ such that $t \neq t^{\prime}$ we have

$$
\left(L^{u, t} \times L^{u+u^{-1}, t}\right)(i, j) \neq\left(L^{u, t^{\prime}} \times L^{u+u^{-1}, t^{\prime}}\right)\left(i^{\prime}, j\right) .
$$

Otherwise we would have

$$
\begin{array}{ll}
i+u j+u t & =i^{\prime}+u j+u t^{\prime}, \\
i+\left(u+u^{-1}\right) j+\left(u+u^{-1}\right) t & =i^{\prime}+\left(u+u^{-1}\right) j+\left(u+u^{-1}\right) t^{\prime},
\end{array}
$$

implying $t=t^{\prime}$, which is a contradiction. As a consequence, the position matrix of the set $\left\{L^{u, t} \times\right.$ $\left.L^{u+u^{-1}, t}: t \in \mathbb{F}_{q}\right\}$ has one unique entry equal to 1 in each column. Considering the rows of this $(0,1)$ matrix as lines and the columns as points, this is equivalent to say that each point belongs to a unique line. Thus, this $(0,1)$-matrix is the incidence matrix of $q^{2}$ parallel lines with $q$ points on each. 
iiii First, let us show that the position matrix of the family $\mathcal{M}=\left\{L^{u, t} \times L^{u+u^{-1}, t}: u, t \in \mathbb{F}_{q}, u \neq 0\right\}$ has $q$ entries equal to 1 in each row and $q-1$ entries equal to 1 in each column. By (ii) the position matrix of each set $\left\{L^{u, t} \times L^{u+u^{-1}, t}: t \in \mathbb{F}_{q}\right\}$ contributes with a unique 1 in each column, yielding that each column of the position matrix of $\mathcal{M}$ has $q-1$ entries equal to 1 . Since each matrix $L^{u, t} \times L^{u+u^{-1}, t}$ has $q^{2}$ distinct entries, then the $q$ position matrices of symbols starting with the same $x$ for any $x \in \mathbb{F}_{q}$ contribute with one unique 1 in each row. So the position matrix of $\mathcal{M}$ has $q$ entries equal to 1 in each row. Therefore, we conclude that the position matrix of $\mathcal{M}$ has order $\left(q^{3}-q^{2}\right) \times q^{3}$ and $q$ entries equal to 1 in each row and $q-1$ entries equal to 1 in each column.

Next, let us show that the girth of $\mathcal{M}$ is at least 6. Otherwise, there exists $u, v \in \mathbb{F}_{q} \backslash\{0\}, u \neq v$ and $i, i^{\prime} j, j^{\prime} \in \mathbb{F}_{q}$ with $j \neq j^{\prime}$ for which

$$
\begin{aligned}
& \left(L^{u, t} \times L^{u+u^{-1}, t}\right)(i, j)=\left(L^{v, t^{\prime}} \times L^{v+v^{-1}, t^{\prime}}\right)\left(i^{\prime}, j\right), \\
& \left(L^{u, t} \times L^{u+u^{-1}, t}\right)\left(i, j^{\prime}\right)=\left(L^{v, t^{\prime}} \times L^{v+v^{-1}, t^{\prime}}\right)\left(i^{\prime}, j^{\prime}\right) .
\end{aligned}
$$

This implies that $L^{u, t}$ and $L^{v, t^{\prime}}$ are not quasi row-disjoint, a contradiction with item (i). Thus the family $\left\{L^{u, t} \times L^{u+u^{-1}, t}: u, t \in \mathbb{F}_{q}, u \neq 0\right\}$ has girth at least 6 . Next let us show that the girth is 8 applying Theorem 2.2. By way of contradiction assume that for three elements $u, v, w$ of $\mathbb{F}_{q} \backslash\{0\}$ there exist three pairwise distinct columns $j, j^{\prime}, j^{\prime \prime}$ for which

$$
\begin{array}{ll}
\left(L^{u, t} \times L^{u+u^{-1}, t}\right)(i, j) & =\left(L^{v, t^{\prime}} \times L^{v+v^{-1}, t^{\prime}}\right)\left(i^{\prime}, j\right) \\
\left(L^{v, t^{\prime}} \times L^{v+v^{-1}, t^{\prime}}\right)\left(i^{\prime}, j^{\prime}\right) & =\left(L^{w, t^{\prime \prime}} \times L^{w+w^{-1}}, t^{\prime \prime}\right)\left(i^{\prime}, j^{\prime}\right) \\
\left(L^{w, t^{\prime \prime}} \times L^{w+w^{-1}}, t^{\prime \prime}\right)\left(i^{\prime \prime}, j^{\prime \prime}\right) & =\left(L^{u, t} \times L^{u+u^{-1}, t}\right)\left(i, j^{\prime \prime}\right) .
\end{array}
$$

Note that $u, v, w$ must be three distinct elements because by (ii) each matrix $L^{u, t} \times L^{u+u^{-1}, t}$ has $q^{2}$ distinct entries. Then from the equality between the first coordinates we have:

$$
\begin{array}{ll}
(u-v) j & =i^{\prime}-i+v t^{\prime}-u t \\
(v-w) j^{\prime} & =i^{\prime \prime}-i^{\prime}+w t^{\prime \prime}-v t^{\prime} \\
(w-u) j^{\prime \prime} & =i-i^{\prime \prime}+u t-w t^{\prime \prime}
\end{array}
$$

Hence

$$
(v-u) j+(w-v) j^{\prime}=(w-u) j^{\prime \prime} .
$$

Moreover, from equalities between the second coordinates in $(6)$ and taking into account $(7)$ we obtain

$$
\left(v^{-1}-u^{-1}\right) j+\left(w^{-1}-v^{-1}\right) j^{\prime}=\left(w^{-1}-u^{-1}\right) j^{\prime \prime} .
$$

Multiplying this equality by uvw we get

$$
w(u-v) j+u(v-w) j^{\prime}=v(u-w) j^{\prime \prime} .
$$

Multiplying (7) by $w$ we also obtain

$$
w(v-u) j+w(w-v) j^{\prime}=w(w-u) j^{\prime \prime} .
$$

Thus adding both equalities 89 and 99 we have

$$
(u-w)(v-w) j^{\prime}=(v-w)(u-w) j^{\prime \prime} .
$$


Taking into account that $u, v, w$ are mutually distinct we get that $j^{\prime}=j^{\prime \prime}$ which is a contradiction. Therefore $\mathcal{M}=\left\{L^{u, t} \times L^{u+u^{-1}, t}: u, t \in \mathbb{F}_{q}, u \neq 0\right\}$ has girth 8 as claimed.

(iv) By (iii) and applying Theorem 2.2. we only need to prove that for all $u, v \in \mathbb{F}_{q} \backslash\{0\}$ with $u \neq v$, any three matrices $L^{u, t} \times L^{u+u^{-1}, t}, L^{v, t} \times L^{v+v^{-1}, t^{\prime}}$ and $\left(t^{\prime \prime} \times[[q-1]]\right) I_{q}$ have girth 8. Otherwise we would have

$$
\begin{array}{lll}
L^{u, t} \times L^{u+u^{-1}, t}(i, j) & =L^{v, t^{\prime}} \times L^{v+v^{-1}}, t^{\prime}\left(i^{\prime}, j\right) \\
L^{v, t^{\prime}} \times L^{v+v^{-1}}, t^{\prime}\left(i^{\prime}, j^{\prime}\right) & \in\left(t^{\prime \prime} \times[[q-1]]\right) I_{q}\left(j^{\prime}, j^{\prime}\right) \\
L^{u, t} \times L^{u+u^{-1}, t}\left(i, j^{\prime}\right) & \in\left(t^{\prime \prime} \times[[q-1]]\right) I_{q}\left(j^{\prime}, j^{\prime}\right)
\end{array}
$$

Then $L^{u, t}(i, j)=L^{v, t^{\prime}}\left(i^{\prime}, j\right)$ and $L^{u, t}\left(i, j^{\prime}\right)=L^{v, t^{\prime}}\left(i^{\prime}, j^{\prime}\right)=t^{\prime \prime}$, meaning that $L^{u, t}$ and $L^{v, t^{\prime}}$ are not quasi row-disjoint, contradicting item $(i)$. Further, by iiii, we know that the position matrix of $\left\{L^{u, t} \times\right.$ $\left.L^{u+u^{-1}, t}: u, t \in \mathbb{F}_{q}, u \neq 0\right\}$ has $q$ ones in each row and $q-1$ ones in each column. Since the rows of the position matrix of $(t \times[[q-1]]) I_{q}$ contributes with one additional one, then the result follows.

To illustrate the method of Theorem 3.1, both the matrices provided by this theorem and their position matrix for the first case $q=2$ are shown in Table 1 . From now on, if there is no confusion an entry $(x, y)$ will be denoted as $x y$. Thus this $(0,1)$-matrix is the incidence matrix of a 2-regular graph of girth 8 , which consists of two cycles of girth 8 . In Table 2 the matrices for $q=3$ are also depicted. The corresponding position matrix is the incidence matrix of a 3-regular graph of girth 8 on 27 vertices in each partite set.

\begin{tabular}{|c|c|c|c|c|c|c|c|c|c|c|}
\hline \multicolumn{3}{|c|}{ MATRICES } & \multicolumn{8}{|c|}{ SYMBOLS } \\
\hline \multirow{3}{*}{$\overline{L^{1,0} \times L^{0,0}}$} & & & & 00 & & 01 & & 10 & 1 & 1 \\
\hline & $\overline{000}$ & $\overline{10}$ & 1 & & 0 & $\overline{0}$ & 0 & 1 & $0 \quad 0$ & \\
\hline & 11 & 01 & 0 & 0 & 0 & 1 & 0 & 0 & 10 & \\
\hline \multirow[t]{2}{*}{$L^{1,1} \times L^{0,1}$} & 10 & $\overline{00}$ & & 1 & 0 & 0 & 1 & 0 & $\overline{0}$ & \\
\hline & 01 & 11 & & 0 & 1 & 0 & 0 & 0 & 0 & \\
\hline \multirow[t]{2}{*}{$(0 \times[1]]]) I_{2}$} & $\{00,01\}$ & 0 & & & 1 & 0 & & 0 & & \\
\hline & 0 & $\{00,01\}$ & 0 & 1 & 0 & 1 & 0 & 0 & $0 \quad 0$ & \\
\hline \multirow{2}{*}{$(1 \times[1]]) I_{2}$} & $\{10,11\}$ & 0 & & 0 & 0 & 0 & 7 & 0 & & \\
\hline & 0 & $\{10,11\}$ & 10 & 0 & 0 & 0 & 0 & 1 & $\begin{array}{ll}0 & 1\end{array}$ & 1 \\
\hline
\end{tabular}
Table 3 contains the matrices for $q=4$. Their position matrix is the incidence matrix of a 4-regular bipartite graph on 64 vertices in each partite set.

Tab. 1: Case $q=2$.

Let us call array of $r$ symbols and $n$ columns the matrix of order $r \times n$

$$
O_{r, n}=\left(\begin{array}{ccc}
1 & \cdots & 1 \\
2 & \cdots & 2 \\
\vdots & \vdots & \vdots \\
r & \cdots & r
\end{array}\right) .
$$

When $r=n$ the array is denoted by $O_{n}$. It is easy to see that the position matrix of $O_{r, n}$ is the incidence matrix of a partial plane consisting in $r$ parallel lines, each one having $n$ points. Using the position matrices of these arrays denoted by $\mathcal{P}\left(O_{r, n}\right)$ and applying Theorem 3.1. we now present the method for constructing the desired $(k, 8)$-bipartite graphs. 


\begin{tabular}{|c|c|c|c|c|c|}
\hline \multicolumn{3}{|c|}{$L^{1, t} \times L^{2, t} \mid L^{2, t} \times L^{1, t}$} & \multicolumn{3}{|c|}{$(t \times[[2]]) I_{3}$} \\
\hline & $0012 \quad 21$ & $0021 \quad 12$ & $\{00,01,02\}$ & 0 & $\overline{0}$ \\
\hline $\mathrm{t}=0$ & $1120 \quad 02$ & $1102 \quad 20$ & 0 & $\{00,01,02\}$ & 0 \\
\hline & $2201 \quad 10$ & $2210 \quad 01$ & 0 & 0 & $\{00,01,02\}$ \\
\hline & 122100 & 211200 & $\{10,11,12\}$ & 0 & 0 \\
\hline$t=1$ & $2002 \quad 11$ & $0220 \quad 11$ & 0 & $\{10,11,12\}$ & \\
\hline & $01 \quad 10 \quad 22$ & $1001 \quad 22$ & 0 & 0 & $\{10,11,12\}$ \\
\hline & $2100 \quad 12$ & $1200 \quad 21$ & $\{20,21,22\}$ & 0 & 0 \\
\hline$t=2$ & $0211 \quad 20$ & $2011 \quad 02$ & 0 & $\{20,21,22\}$ & 0 \\
\hline & $1022 \quad 01$ & $0122 \quad 10$ & 0 & 0 & $\{20,21,22\}$ \\
\hline
\end{tabular}

Tab. 2: Matrices for the case $q=3$.

\begin{tabular}{|c|c|c|c|c|c|}
\hline & \begin{tabular}{c|c|c}
$L^{1, t} \times L^{0, t}$ & $L^{2, t} \times L^{1, t}$ & $L^{3, t} \times L^{1, t}$
\end{tabular} & & $(t \times[$ & $[3] 1) I_{4}$ & \\
\hline & \begin{tabular}{lllllllll|llll}
00 & 10 & 20 & 30 & 0 & 21 & 32 & 13 & 0 & 31 & 12 & 23 \\
\end{tabular} & $\{00,01,02,03\}$ & 0 & 0 & 0 \\
\hline & 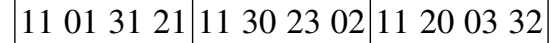 & 0 & $\{00,01,02,03\}$ & 0 & 0 \\
\hline$t=0$ & 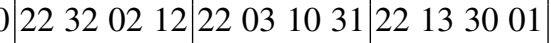 & 0 & 0 & $\{00,01,02,03\}$ & 0 \\
\hline & 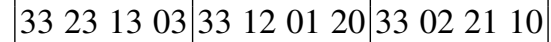 & 0 & 0 & 0 & $\{00,01,02,03\}$ \\
\hline & 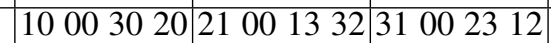 & $\{10,11,12,13\}$ & 0 & 0 & 0 \\
\hline$t=1$ & 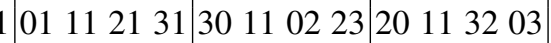 & 0 & $\{10,11,12,13\}$ & 0 & 0 \\
\hline & 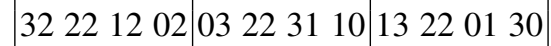 & 0 & 0 & $\{10,11,12,13\}$ & 0 \\
\hline & 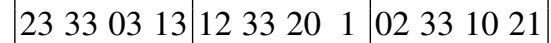 & 0 & 0 & 0 & $\{10,11,12,13\}$ \\
\hline & \begin{tabular}{llllllll|llll}
20 & 30 & 00 & 10 & 32 & 13 & 00 & 21 & 12 & 23 & 0 & 31
\end{tabular} & $\{20,21,22,23\}$ & 0 & 0 & 0 \\
\hline & 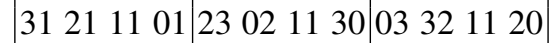 & 0 & $\{20,21,22,23\}$ & 0 & 0 \\
\hline$t=2$ & 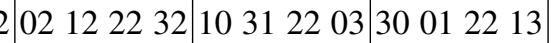 & 0 & 0 & $\{20,21,22,23\}$ & 0 \\
\hline & 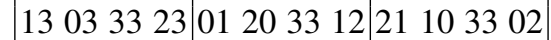 & 0 & 0 & 0 & $\{20,21,22,23\}$ \\
\hline & 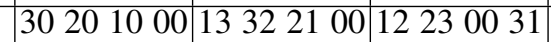 & $\{30,31,32,33\}$ & 0 & 0 & 0 \\
\hline$t=3$ & 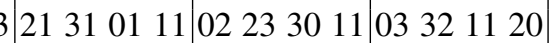 & 0 & $\{30,31,32,33\}$ & 0 & 0 \\
\hline & 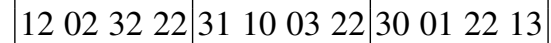 & 0 & 0 & $\{30,31,32,33\}$ & 0 \\
\hline & 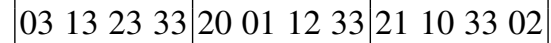 & 0 & 0 & 0 & $\{30,31,32,33\}$ \\
\hline
\end{tabular}

Tab. 3: Matrices for the case $q=4$.

Theorem 3.2 Let $q$ be a power prime, and let $\mathbb{F}_{q}$ be the Galois field of order $q$. For each $u, t \in \mathbb{F}_{q}, u \neq 0$, let $L^{u, t}$ be the matrix $L^{u, t}(i, j)=i+u j+u t, i, j \in \mathbb{F}_{q}$, and let $M$ be the position matrix of the family

$$
\left\{L^{u, t} \times L^{u+u^{-1}, t}: u, t \in \mathbb{F}_{q}, u \neq 0\right\} \cup\left\{(t \times[[q-1]]) I_{q}: t \in \mathbb{F}_{q}\right\} .
$$

Then the following assertions hold:

(i) The (0,1)-matrix

$$
\begin{array}{|l|l|}
\hline M & \mathcal{P}\left(O_{q^{2}, q}\right)^{\top} \\
\hline
\end{array}
$$

is the incidence matrix of a bipartite graph of girth 8 with $q^{3}+q^{2}$ vertices in one partite set having degree $q+1$, and $q^{3}$ vertices in the other partite set having degree $q$. 
(ii) Let $\left(L^{u, t} \times L^{u+u^{-1}, t}\right)_{0}$ denote the matrix obtained from $L^{u, t} \times L^{u+u^{-1}, t}$ by replacing each entry $0 x$ with 0 for all $x \in \mathbb{F}_{q}$. Let $M_{0}$ be the position matrix of the family

$$
\left\{\left(L^{u, t} \times L^{u+u^{-1}, t}\right)_{0}: u, t \in \mathbb{F}_{q}, u \neq 0\right\} \cup\left\{(t \times[[q-1]]) I_{q}: t \in \mathbb{F}_{q}, t \neq 0\right\} .
$$

Then

$$
\begin{array}{|c|c|}
\hline M_{0} & \mathcal{P}\left(O_{q^{2}-q, q}\right)^{\top} \\
\cline { 2 - 2 } & {[0]} \\
\hline
\end{array}
$$

is the incidence matrix of a q-regular bipartite graph of girth 8 with $q^{3}-q$ vertices in each partite set.

(iii) Let $k$ be an integer such that $3 \leq k \leq q-1$ and let $\left(L^{u, t} \times L^{u+u^{-1}, t}\right)_{q-k}$ denote the matrix obtained from $L^{u, t} \times L^{u+u^{-1}, t}$ by replacing with 0 the entries $0 y$ and $(x, x+s)$ for all $x, y, s \in \mathbb{F}_{q}$ for $s=0, \ldots, q-1-k$. Let $M_{q-k}$ be the position matrix of

$$
\left\{\left(L^{u, t} \times L^{u+u^{-1}, t}\right)_{q-k}: u=1, \ldots, k-1\right\} \cup\left\{(t \times([[q-1]] \backslash\{t, t+1, \ldots, t+q-1-k\})) I_{q}: t \neq 0\right\} .
$$

Suppose that the $q$ columns of $O_{k q-q, q}$ are indexed by $j \in \mathbb{F}_{q}$. Let $O_{k q-q, q}^{*}$ be the matrix obtained from $O_{k q-q, q}$ by changing for 0 the entries $\left(i,-u^{2} s\right)$ for all $u=1, \ldots, k-1, i=(u-1) q+1, \ldots, u q$ and $s=0, \ldots, q-1-k$. Then

$$
\begin{array}{|c|c|}
\hline M_{q-k} & \mathcal{P}\left(O_{k q-q, q}^{*}\right)^{\top} \\
\cline { 2 - 2 } & {[0]} \\
\hline
\end{array}
$$

is the incidence matrix of a $k$-regular bipartite graph of girth 8 with $k q^{2}-q$ vertices in each partite set.

Proof: (i) From Theorem 3.1, it follows that $M$ is the incidence matrix of a bipartite graph of girth 8 with $q^{3}$ lines and $q^{3}$ points. Thus we need to prove that the $q^{2}$ columns of $\mathcal{P}\left(O_{q^{2}, q}\right)^{\top}$ can be added to $M$ without decreasing the girth 8 . It is readily seen that after adding these columns, the girth is at least 6, because by Theorem 3.1 for each $u \in \mathbb{F}_{q} \backslash\{0\}$ the set $\left\{L^{u, t} \times L^{u+u^{-1}, t}: t \in \mathbb{F}_{q},\right\}$ consists of $q^{2}$ parallel lines. Thus suppose that $[10]$ contains as a sub-matrix the incidence matrix of a cycle of length 6 . Then there exists $u, v \in \mathbb{F}_{q} \backslash\{0\}$ and $i, i^{\prime}, i^{\prime \prime}, j, j^{\prime} \in \mathbb{F}_{q}$ with $u \neq v, i \neq i^{\prime}$ and $j \neq j^{\prime}$ such that

$$
\begin{aligned}
& \left(L^{u, t} \times L^{u+u^{-1}, t}\right)(i, j)=\left(L^{v, t^{\prime}} \times L^{v+v^{-1}, t^{\prime}}\right)\left(i^{\prime \prime}, j\right), \\
& \left(L^{u, t} \times L^{u+u^{-1}, t}\right)\left(i^{\prime}, j^{\prime}\right)=\left(L^{v, t^{\prime}} \times L^{v+v^{-1}, t^{\prime}}\right)\left(i^{\prime \prime}, j^{\prime}\right) .
\end{aligned}
$$

From the equalities between their coordinates we obtain

$$
\begin{array}{ll}
i-i^{\prime}+u\left(j-j^{\prime}\right) & =v\left(j-j^{\prime}\right) \\
i-i^{\prime}+\left(u+u^{-1}\right)\left(j-j^{\prime}\right) & =\left(v+v^{-1}\right)\left(j-j^{\prime}\right) .
\end{array}
$$

Hence $u^{-1}\left(j-j^{\prime}\right)=v^{-1}\left(j-j^{\prime}\right)$, implying $u=v$ or $j=j^{\prime}$, a contradiction in either case. Further if $\left(L^{u, t} \times L^{u+u^{-1}, t}\right)(i, j) \in\left(t^{\prime} \times[[q-1]] I_{q}\right)\left(i^{\prime \prime}, j\right)$, and $\left(L^{u, t} \times L^{u+u^{-1}, t}\right)\left(i^{\prime}, j^{\prime}\right) \in\left(t^{\prime} \times[[q-1]] I_{q}\right)\left(i^{\prime \prime}, j^{\prime}\right)$, then then $i^{\prime \prime}=j=j^{\prime}$ which is also a contradiction. 


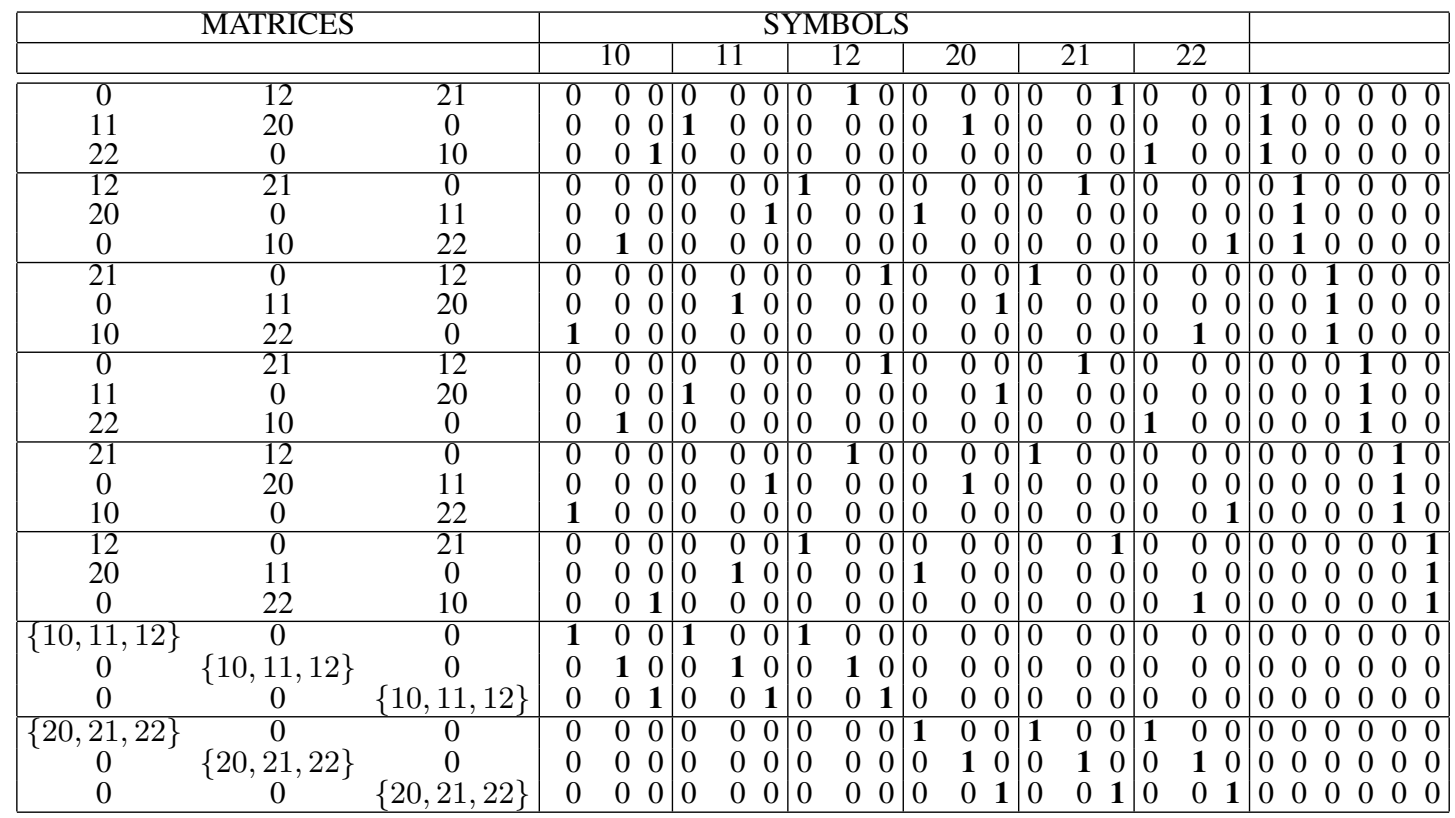

Tab. 4: Case $q=3$ for (iii) of Theorem 3.2 Incidence matrix of a (3,8)-graph on 48 vertices.

(iii) This item follows directly from the fact that $M_{0}$ is a sub-matrix of $M$ obtained by deleting the first $q^{2}$ columns, which correspond to the position of the symbols starting by 0 . Hence $M_{0}$ has $q^{3}-q^{2}$ columns. Moreover, the total number of rows of $M_{0}$ is the number of matrices $L^{u, t} \times L^{u+u^{-1}, t}$, (that is, $(q-1) q)$ plus the number of matrices $(t \times([[q-1]])) I_{q}, t \neq 0$, (a total of $\left.q-1\right)$ multiplied by $q$, that is, $q\left(q^{2}-q+q-1\right)=q^{3}-q$. Thus $M_{0}$ is a matrix of order $\left(q^{3}-q\right) \times\left(q^{3}-q^{2}\right)$. Since $\mathcal{P}\left(O_{q^{2}-q . q}\right)^{\top}$ contributes with $q^{2}-q$ more columns, then $(11)$ is a square matrix of order $q^{3}-q$. Reasoning as in (iii) of Theorem 3.1. we obtain that 11 is the incidence matrix of a bipartite graph of girth 8 , which has $q^{3}-q$ columns and $q^{3}-q$ rows both having $q$ ones, so this item is valid. By way of example, both the matrices provided by this item (iii) and their position matrix for the case $q=3$ are shown in Table 4 . Thus this $(0,1)$-matrix is the incidence matrix of a 3-regular graph of girth 8 on 24 vertices in each partite set.

(iii) Note that (12) is a sub-matrix of (10), then it is the incidence matrix of a bipartite graph of girth 8 . Moreover, $M_{q-k}$ is obtained from $M$ by deleting the first $q^{2}$ columns which corresponds to the position matrix of symbols starting by 0 , and by deleting also $(q-k)(q-1) q$ columns corresponding to the symbols $(x+s, x+2 s)$ for all $s=0,1, \ldots, q-1-k$ which have been changed for 0 . Then the total number of columns of $M_{q-k}$ is

$$
q^{3}-q^{2}-(q-k)(q-1) q=k q(q-1) .
$$

The total number of rows of $M_{q-k}$ is given by the number of matrices $\left(L^{u, t} \times L^{u+u^{-1}, t}\right)_{q-k}, u=$ $1, \ldots, k-1, t \in \mathbb{F}_{q}$, plus the number of matrices $(t \times([[q-1]] \backslash\{t, t+1, \ldots, t+q-1-k\})) I_{q}: t \neq 0$, $t \neq 0$, (a total of $q-1$ ) multiplied by $q$, that is

$$
(k-1) q^{2}+(q-1) q=k q^{2}-q .
$$


Thus $M_{q-k}$ is a matrix of order $\left(k q^{2}-q\right) \times\left(k q^{2}-k q\right)$. Since $\mathcal{P}\left(O_{k q-q, q}^{*}\right)^{\top}$ contributes with $k q-q$ more columns, then 12 is a square matrix of order $k q^{2}-q$.

To finish the proof of this item, we only need to show that 12 has $k$ ones in each row and $k$ ones in each column. To see this, let us show that given a fixed $s=0,1, \ldots, q-1-k$, the entries $(y, y+s)$ for all $y \in \mathbb{F}_{q}$, are in the same column of each matrix $L^{u, t} \times L^{u+u^{-1}, t}$. Suppose $L^{u, t} \times L^{u+u^{-1}, t}(i, j)=$ $(y, y+s)$, that is

$$
i+u j+u t=y, \text { and } i+\left(u+u^{-1}\right) j+\left(u+u^{-1}\right) t=y+s .
$$

Then $u^{-1}(j+t)=s$, which implies $j=u s-t$. Thus our claim is true since the symbols $(y, y+s)$ are placed in the same column $u s-t$ of the matrix $L^{u, t} \times L^{u+u^{-1}, t}$. Therefore, after changing for 0 the symbols $0 x$ and $(y, y+s)$ for all $x, y \in \mathbb{F}_{q}$ and for all $s=0, \ldots, q-1-k$ we obtain a new matrix having $k$ entries different from 0 in the rows $i$ such that $i+u^{2} s=0$, and in the remaining rows $k-1$ entries different from 0 . Since in the rows $i$ of $\mathcal{P}\left(O_{q k-q, q}^{*}\right)^{\top}$ such that $i+u^{2} s \neq 0$ there is a 1 by hypothesis, then $\mathcal{P}\left(O_{q k-q, q}^{*}\right)^{\top}$ contributes with one additional entry equal to 1 in the same rows as those having $k-1$ entries different from 0 . Hence 12 is a square matrix of order $k q^{2}-q$ having $k$ ones in each row and clearly in each column.

By way of example, both the matrices provided by this item (iii) and their position matrix for the case $q=4$ and $k=3$ are shown in Table 5 , and the corresponding matrices for $q=5$ and $k=3$ are shown in Table 6. Thus, these $(0,1)$-matrices are the incidence matrix of a 3-regular graph of girth 8 on 44 vertices in each partite set for $q=4$ and 70 vertices in each partite set for $q=5$.

\section{Conclusion}

For $q$ a prime power and $3 \leq k \leq q$ we have presented a method providing the incidence matrices of $k$-regular bipartite graphs of girth 8 with $k q^{2}-q$ vertices in each partite set. Thus if $n(k, 8)$ denotes the order of a $(k, 8)$-cage, it follows from (1) that

$$
2 k\left(k^{2}-2 k+2\right) \leq n(k, 8) \leq 2 q(k q-1) .
$$

Hence the $q$-regular bipartite graphs constructed in this work have an excess of $4 q^{2}-6 q$. And the $(q-1)$ regular bipartite graphs have an excess of $8 q^{2}-20 q+10$.

As regards to known upper bounds on $n(k, g)$, Lazebnik, Ustimenko and Woldar [19] gave the following result: Let $k \geq 2$ and $g \geq 5$ be integers, and let $q$ denote the smallest odd prime power for which $k \leq q$. Then,

$$
n(k, g) \leq 2 k q^{\frac{3}{4} g-a},
$$

where $a=4,11 / 4,7 / 2,13 / 4$ for $g \equiv 0,1,2,3(\bmod 4)$, respectively. According to $\sqrt{13}, n(k, 8) \leq 2 k q^{2}$, therefore the graphs provided by our method also improve this result for $g=8$. A construction giving this upper bound (13) for $g=8$ appeared for the first time in [18] and was used later in [14] and probably, the simplest exposition of it is in Section 2.4 of [20]. In [15], $(q, 8)$-graphs with $2 q\left(q^{2}-2\right)$ vertices were constructed using geometrical techniques. But for regularities $k<q$ the graphs constructed in this paper have the smallest number of vertices among the known regular graphs with girth 8 . 


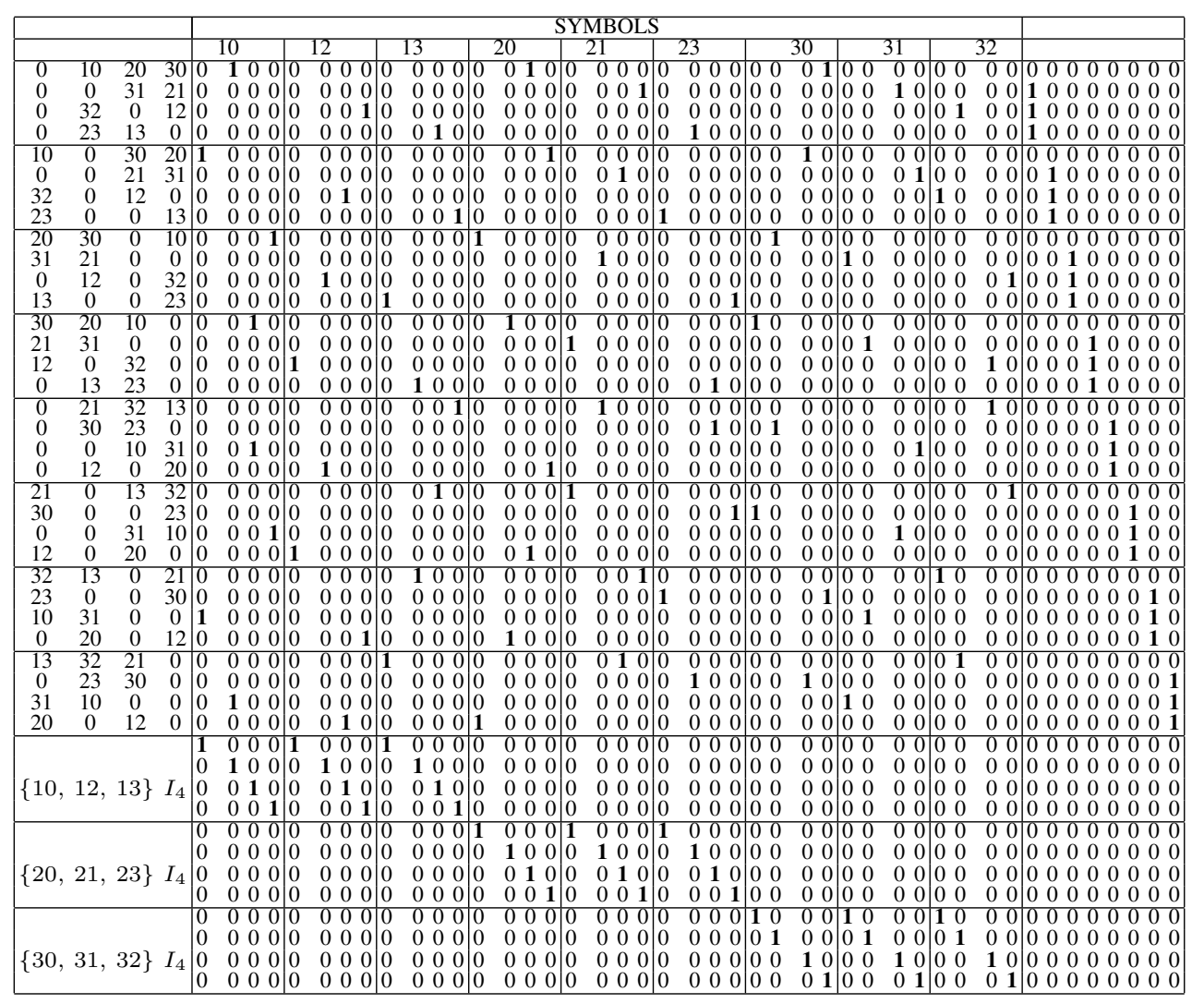

Tab. 5: Case $q=4$ for $k=3$ of Theorem 3.2 (iii). Incidence matrix of a (3,8)-graph on 88 vertices.

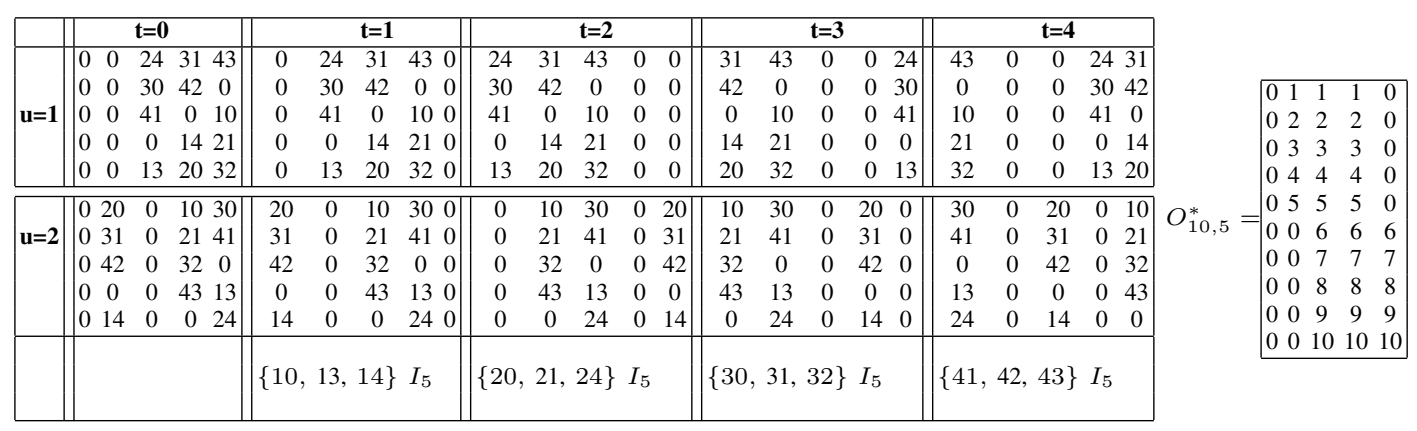

Tab. 6: Matrices for $q=5$ and $k=3$ according to Case (iii) of Theorem 3.2 


\section{Acknowledgements}

I would like to thank the anonymous referees for their suggestions and for their valuable comments.

\section{References}

[1] M. Abreu, M. Funk, D. Labbate, V. Napolitano, On (minimal) regular graph of girth 6, Australasian Journal of Combinatorics 35 (2006), 119-132.

[2] G. Araujo, D. González Moreno, J.J. Montellano, O. Serra, On upper bounds and conectivity of cages, Australasian Journal of Combinatorics 38 (2007), 221-228.

[3] C. BALBUENA, Incidence matrices of projective planes and other bipartite graphs of few vertices, Siam Journal of Discrete Maths. 22(4) (2008), 1351-1363.

[4] E. Bannai And T. Ito, On finite Moore graphs, J. Fac. Sci. Univ. Tokyo, Sect. I A Math 20 (1973), 191-208.

[5] L.M. Batten, Combinatorics of finite geometries, Cambridge University Press, Cambridge, UK, 1997.

[6] C.T. Benson, Minimal regular graphs of girth eight and twelve, Canad. J. Math 18 (1966), 10911094.

[7] N. BIgGS, Algebraic Graph Theory, Cambridge University Press, New York, 1996.

[8] N. BIGGS, Construction for cubic graphs with large girth, Electron. J. Combin. 5 (1998), \#A1.

[9] G. BRINKMANn, B. D. MCKAY AND C. SAAGER, The smallest cubic graphs of girth nine, Combinatorics Probability and Computing 5, (1995), 1-13.

[10] P. ERdöS AND H. SACHS, Reguläre Graphen gegebener Taillenweite mit minimaler Knotenzahl, Wiss. Z. Uni. Halle (Math. Nat.), 12 (1963), 251-257.

[11] G. Exoo, A Simple Method for Constructing Small Cubic Graphs of Girths 14, 15 and 16, Electron. J. Combin. 3 (1996).

[12] G. Exoo And R. JajCAY, Dynamic Cage Survey, Electron. J. Combin. 15 (2008) \#DS16.

[13] W. Feit And G. Higman, The non-existence of certain generalized polygons, J. Algebra 1 (1964), 114-131.

[14] Z. Furedi, F. Lazebnik, A. Seress, V.A. Ustimenko and J.A. Woldar, Graphs of prescribed girth and bi-degree, J. of Combin. Theory Series B 64(2) (1995), 228-239.

[15] A. GÁCS And T. HÉGER, On geometric constructions of $(k, g)$-graphs, Contributions to Discrete Mathematics, 3(1) (2008) 63-80.

[16] C. Godsil And G. RoYLe, Algebraic Graph Theory, Springer, NY 2000. 
[17] D.A. Holton and J. Sheehan, The Petersen Graph, Chapter 6: Cages, Cambridge University (1993).

[18] F. LAZEBnik, V.A. Ustimenko, AND A.J. WoldAR, New constructions of bipartite graphs on $m, n$ vertices with may edges and without small cycles, J. of Combin. Theory Series B 61(1) (1994), 111-117.

[19] F. LAZEBnik, V.A. Ustimenko, AND A.J. WolDAR, New upper bounds on the order of cages, Electron. J. Combin. 4 (1997), no. 2.

[20] F. LAZEBNIK, AND A.J. WolDAR, General properties of some families of graphs defined by systems of equations, J. Graph Theory 38 (2001), 65-86.

[21] J. H. van LinT AND R. M. Wilson, A course in Combinatorics, Cambridge University Press, UK 1994.

[22] M. MERINGER, Fast generation of regular graphs and construction of cages, J. Graph Theory 30 (1999), 137-146.

[23] M. O' KeEFE AND P.K. Wong, A smallest graph of girth 10 and valency 3, J. of Combin. Theory Series B 29 (1980), 91-105.

[24] M. O' KeEfE And P.K. Wong, The smallest graph of girth 6 and valency 7, J. Graph Theory 5 (1981), no. 1, 79-85.

[25] T. Pisanski, M. Boben, D. Marusic, A. Orbanic, A. Graovac, The 10-cages and derived configurations, Discrete Mathematics 275 (2004), 265-276.

[26] G. RoYLE, http://www.cs.uwa.edu.au/ gordon/data.html.

[27] W. T. TUTTE, A family of cubical graphs, Proc. Cambridge Philos. Soc. (1947), 459-474.

[28] P.K. Wong, A regular graph of girth 6 and valency 11, Internat. J. Math. Sci 9, (1986), 561-565.

[29] P. K. Wong, Cages-a survey, J. Graph Theory 6 (1982), 1-22. 